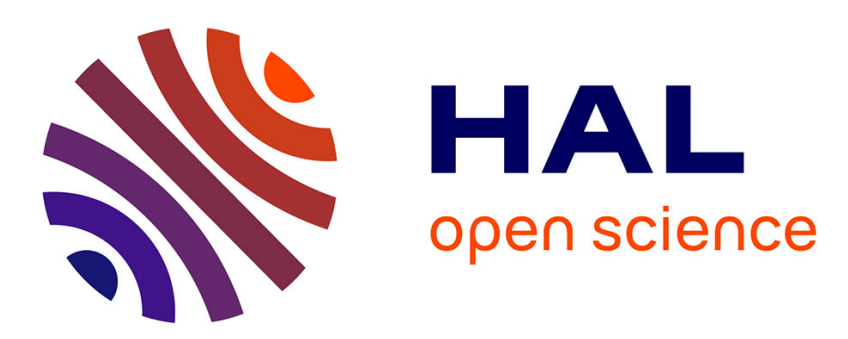

\title{
Computing divisors and common multiples of quasi-linear ordinary differential equations
}

\author{
Dima Grigoriev, Fritz Schwarz
}

\section{To cite this version:}

Dima Grigoriev, Fritz Schwarz. Computing divisors and common multiples of quasi-linear ordinary differential equations. Lecture Notes in Computer Science, 2013. hal-03044754

\section{HAL Id: hal-03044754 \\ https://hal.science/hal-03044754}

Submitted on 7 Dec 2020

HAL is a multi-disciplinary open access archive for the deposit and dissemination of scientific research documents, whether they are published or not. The documents may come from teaching and research institutions in France or abroad, or from public or private research centers.
L'archive ouverte pluridisciplinaire HAL, est destinée au dépôt et à la diffusion de documents scientifiques de niveau recherche, publiés ou non, émanant des établissements d'enseignement et de recherche français ou étrangers, des laboratoires publics ou privés. 


\title{
Computing divisors and common multiples of quasi-linear ordinary differential equations
}

\author{
Dima Grigoriev \\ CNRS, Mathématiques, Université de Lille \\ Villeneuve d'Ascq, 59655, France \\ Dmitry.Grigoryev@math .univ-lille1.fr \\ http://en.wikipedia.org/wiki/Dima_Grigoriev
}

\author{
Fritz Schwarz \\ Fraunhofer Gesellschaft, Institut SCAI \\ 53754 Sankt Augustin, Germany \\ fritz.schwarz@scai.fraunhofer.de \\ www.scai.fraunhofer.de/schwarz.html
}

\begin{abstract}
If solutions of a non-linear differential equation are contained in solutions of another equation we say that the former equation is a generalized divisor of the latter one. We design an algorithm which finds first-order quasi-linear generalized divisors of a second-order quasi-linear ordinary differential equation. If solutions of an equation contain solutions of a pair of equations we say that the equation is a common multiple of the pair. We prove that a quasi-linear common multiple of a pair of quasi-linear equations always exists and design an algorithm which yields a quasilinear common multiple.
\end{abstract}

\section{Introduction}

The problem of factoring linear ordinary differential operators $L=T \circ Q$ was studied in [10]. Algorithms for this problem were designed in [3], [11] (in [3] a complexity bound better than for the algorithm from [10] was established). In [5] an algorithm is exhibited for factoring a partial linear differential operator in two variables with a separable symbol. In [4] an algorithm is constructed for finding all the first-order factors of a partial linear differential operator in two variables. A generalization of factoring for $D$-modules (in other words, for systems of linear partial differential operators) was considered in [6]. A particular case of factoring for $D$-modules is the Laplace problem [2], [14] (a short exposition of the Laplace problem one can find in [7]).

The meaning of factoring for search of solutions is that any solution of operator $Q$ is a solution of operator $L$, thus factoring allows one to diminish the order of operators.

Much less is known for factoring non-linear (even ordinary) differential equations. In Section 1 we design an algorithm for finding (first-order) generalized divisors of a secondorder quasi-linear differential equation. 
We note that our definition of generalized divisors is in the frames of differential ideals [9], rather than the definition of factorization from [13], [1] being in terms of a composition of nonlinear ordinary differential polynomials. In [1] a decomposition algorithm is designed.

One can also introduce a different (from [13], [1]) concept of composition (which yields generalized divisors) as follows. For a differential field $K$ consider an operator $A=\sum_{0 \leq i \leq n} a_{i} \cdot \frac{d^{i}}{d x^{i}}$ acting on the algebra $K\{y\}$ of differential polynomials in $y$ [9] where the coefficients $a_{i} \in K\{y\}$, the result of the action we denote by $A * z \in K\{y\}$ for $z \in K\{y\}$. Clearly, $z$ is a generalized divisor of $A * z$. In Section 1 we show, conversely, that if a quasilinear $z \in K\{y\}$ is a generalized divisor of $v \in K\{y\}$ then $v=A * z$ for an appropriate operator $A$.

Easy examples demonstrate that the two considered compositions differ from each other. In the sense of [13], [1] we have $1=1 \circ z$ for an arbitrary $z \in K\{y\}$, while 1 cannot be represented as $A * y$. On the other hand, $y \cdot y^{\prime}=y * y^{\prime}$, while one cannot represent $y \cdot y^{\prime}$ as $g \circ y^{\prime}$ for any $g \in K\{y\}$.

In Section 2 we define a common multiple of a pair of equations as an equation to which satisfy solutions of both equations. We prove the existence of a quasi-linear common multiple for any pair of quasi-linear differential equations, design an algorithm for computing a quasi-linear common multiple and bound its complexity in terms of Grzegorczyk's hierarchy of primitive-recursive functions.

It would be interesting to extend the algorithm from Section 1 to equations of arbitrary orders and from quasi-linear to arbitrary non-linear ordinary equations (then, perhaps, to partial differential equations).

\section{A bound of the degree and an algorithm for gener- alized divisors}

We study second-order non-linear ordinary differential equations of the form

$$
y^{\prime \prime}=f\left(y^{\prime}, y, x\right)
$$

for a polynomial $f \in \overline{\mathbb{Q}}[z, y, x]$. We assume the coefficients of polynomials to be algebraic since we are interested in algorithms, although for the purposes of bounds (see below) one can consider coefficients from an arbitrary field with characteristics zero.

Definition 1.1 We say that a first-order equation

$$
y^{\prime}=p(y, x)
$$

is a generalized divisor of (1), where $p \in \overline{\mathbb{Q}}[y, x]$, if any y satisfying (2), is a solution of (1).

It suffices to verify the condition in the definition just for generic $y$ [9], i. e. $y$ satisfying only the differential polynomials from the differential ideal generated by $y^{\prime}-p(y, x)$. In particular, $y$ is algebraically independent of $x$ over $\mathbb{Q}$. 
Lemma 1.2 If (2) is a generalized divisor of (1) then the differential polynomial $y^{\prime \prime}-f\left(y^{\prime}, y, x\right)$ has a form $A *\left(y^{\prime}-p(y, x)\right)$ for an operator $A=\frac{d}{d x}+a_{0}$ with a suitable differential polynomial $a_{0} \in \overline{\mathbb{Q}}\left[y^{\prime}, y, x\right]$.

The converse statement is evident.

Proof. Dividing with remainder (with respect to $y^{\prime}$ ) the differential polynomial

$$
u:=y^{\prime \prime}-f\left(y^{\prime}, y, x\right)-\left(y^{\prime}-p(y, x)\right)^{\prime} \in \overline{\mathbb{Q}}\left[y^{\prime}, y, x\right]
$$

by $y^{\prime}-p(y, x)$, we get a differential polynomial $a_{0} \in \overline{\mathbb{Q}}\left[y^{\prime}, y, x\right]$ such that $u=a_{0} \cdot\left(y^{\prime}-\right.$ $p(y, x))+v$ for suitable $v \in \overline{\mathbb{Q}}[y, x]$. Any solution $y$ of $(2)$ is a solution of $v$, hence $v \equiv 0$ since $y$ is algebraically independent with $x$.

Remark 1.3 i) The proof of Lemma 1.2 provides an algorithm to test whether (2) is a generalized divisor of (1).

ii) Lemma 1.2 holds for an arbitrary quasi-linear differential polynomial of the form $y^{(n)}-p_{n}\left(y^{(n-1)}, \ldots, y, x\right)$ in place of (2) and for an arbitrary differential polynomial (not necessary quasi-linear) in place of (1).

From (2) we have

$$
y^{\prime \prime}=\frac{\partial p}{\partial y} \cdot p+\frac{\partial p}{\partial x}
$$

Substituting this into (1) and rewriting

$$
f\left(y^{\prime}, y, x\right)=\sum_{0 \leq i \leq l} f_{i} \cdot\left(y^{\prime}\right)^{i}
$$

where $f_{i} \in \overline{\mathbb{Q}}[y, x]$, we get

$$
\frac{\partial p}{\partial y} \cdot p+\frac{\partial p}{\partial x}=\sum_{0 \leq i \leq l} f_{i} \cdot p^{i}
$$

Observe that (4) is equivalent to that (2) is a generalized divisor of (1).

Then (4) implies that

$$
p \mid\left(f_{0}-\frac{\partial p}{\partial x}\right)
$$

Hence either $\operatorname{deg}_{x} p \leq \operatorname{deg}_{x} f_{0}$ or $f_{0}=\frac{\partial p}{\partial x}$. Indeed, expand $p=\sum_{0 \leq j \leq k} a_{j} \cdot x^{j}$ for certain polynomials $a_{j} \in \overline{\mathbb{Q}}[y], a_{k} \neq 0$. If $k=\operatorname{deg}_{x} p>\operatorname{deg}_{x} f_{0}$ then $\operatorname{deg}\left(f_{0}-\frac{\partial p}{\partial x}\right)<k$, therefore $f_{0}=\frac{\partial p}{\partial x}$ due to $(5)$.

In a similar way, we claim that either $\operatorname{deg}_{y} p \leq \operatorname{deg}_{y} f_{0}$ or $f_{0}=\frac{\partial p}{\partial x}$. Indeed, expand $p=\sum_{0 \leq i \leq m} b_{i} \cdot y^{i}$ for certain polynomials $b_{i} \in \overline{\mathbb{Q}}[x], b_{m} \neq 0$. If $m=\operatorname{deg}_{y} p>\operatorname{deg}_{y} f_{0}$ then the coefficient of $f_{0}-\frac{\partial p}{\partial x}$ at monomial $y^{m}$ equals $-\frac{\partial b_{m}}{\partial x}$. If $\frac{\partial b_{m}}{\partial x} \neq 0$, and thereby $y^{m}$ is the 
leading monomial of $f_{0}-\frac{\partial p}{\partial x}$ with respect to the expansion in $y$, we get a contradiction with (5). Therefore, $\frac{\partial b_{m}}{\partial x}=0$ and $f_{0}=\frac{\partial p}{\partial x}$ due to (5), which proves the claim.

So, it remains to consider the case $f_{0}=\frac{\partial p}{\partial x}$. Then (4) entails that

$$
\frac{\partial p}{\partial y}=\sum_{0 \leq i \leq l-1} f_{i+1} \cdot p^{i}
$$

hence $p \mid\left(f_{1}-\frac{\partial p}{\partial y}\right)$. Arguing as above, we deduce that either $\operatorname{deg}_{x} p \leq \operatorname{deg}_{x} f_{1}, \operatorname{deg}_{y} p \leq$ $\operatorname{deg}_{y} f_{1}$ or $f_{1}=\frac{\partial p}{\partial y}$.

We note that in the latter case $f_{1}=\frac{\partial p}{\partial y}, f_{0}=\frac{\partial p}{\partial x}$ (whence $\sum_{2 \leq i \leq l} f_{i} \cdot p^{i-2}=0$ because of (4)), and $p$ is determined uniquely up to an additive constant. Moreover, in this case $\operatorname{deg}_{x} p \leq 1+\operatorname{deg}_{x} f_{0}, \operatorname{deg}_{y} p \leq 1+\operatorname{deg}_{y} f_{1}$.

Summarizing, we conclude with the following theorem.

Theorem 1.4 i) If (2) is a generalized divisor of (1) then either $\operatorname{deg}_{x} p \leq \operatorname{deg}_{x} f, \operatorname{deg}_{y} p \leq$ $\operatorname{deg}_{y} f$ or $f_{1}=\frac{\partial p}{\partial y}, f_{0}=\frac{\partial p}{\partial x}$, see (3) (which determines $p$ up to an additive constant), and in the latter case $\operatorname{deg}_{x} p \leq 1+\operatorname{deg}_{x} f_{0}, \operatorname{deg}_{y} p \leq 1+\operatorname{deg}_{y} f_{1}$.

ii) An algorithm which either constructs a generalized divisor (2) of (1) or finds out that it does not exist, looks for polynomial $p$ with indeterminate coefficients from $\bar{Q}$ satisfying the degree bounds

$$
\operatorname{deg}_{x} p \leq \max \left\{\operatorname{deg}_{x} f, 1+\operatorname{deg}_{x} f_{0}\right\}, \quad \operatorname{deg}_{y} p \leq \max \left\{\operatorname{deg}_{y} f, 1+\operatorname{deg}_{y} f_{1}\right\}
$$

from item i), solving (4) as a system of polynomial equations in the indeterminate coeffcients of $p$.

Example 1 Consider the equation

$$
E \equiv y^{\prime \prime}+(x+3 y) y^{\prime}+y^{3}+x y^{2}=0 .
$$

According to the above theorem $\operatorname{deg}_{x} E=1$ and $\operatorname{deg}_{y} E=3$, i.e. $\operatorname{deg}_{x} p \leq 1$ and $\operatorname{deg}_{y} p \leq 3$. The second alternative $\frac{\partial p}{\partial x}=f_{0}=-x y^{2}-y^{3}, \frac{\partial p}{\partial y}=f_{0}=-x-3 y$ does not apply because this system for $p$ is inconsistent. Proceeding as described above, two divisors are obtained and the representations

$$
E \equiv\left(y^{\prime}+y^{2}\right)^{\prime}+(y+x)\left(y^{\prime}+y^{2}\right) \quad \text { and } \quad E=\left(y^{\prime}+y^{2}+x y-1\right)^{\prime}+y\left(y^{\prime}+y^{2}+x y-1\right)
$$

follow. They yield the two one-parameter solutions

$$
y=\frac{1}{x+C} \quad \text { and } \quad y=\frac{1}{x}+\frac{1}{x^{2}} \frac{\exp \left(-\frac{1}{2} x^{2}\right)}{\int \exp \left(-\frac{1}{2} x^{2}\right) \frac{d x}{x^{2}}+C}
$$

respectively.

An extension of the definition of a generalized divisor is the definition of a first integral. 
Definition 1.5 We say that $y^{\prime}-p(y, x)$ is a first integral of (1) if for any constant $c$ any solution $y$ of equation $y^{\prime}-p(y, x)=c$ is also a solution of (1).

Denote by $A(c)$ a formula obtained from (4) by means of replacing $p$ with $p+c$. Arguing as above, we get that $y-p(y, x)$ is a first integral of (1) iff $A(c)$ holds for any constant $c$. We obtain the same bound on $\operatorname{deg} p$ as in Theorem i). The algorithm for finding first integrals applies a quantifier elimination procedure to the following formula of the first-order theory of algebraically closed fields:

$$
\exists P \forall c A(c)
$$

where $P$ denotes the vector of indeterminate coefficients of polynomial $p$. Thus, the algorithm finds the constructible set of all vectors $P$ for which $\forall c A(c)$ holds. These vectors $P$ provide all first integrals of (1).

\section{Computing common multiples of quasi-linear dif- ferential equations}

Definition 2.1 We say that a differential equation $f=0$ is a common multiple of equations $f_{1}=0$ and $f_{2}=0$ if solutions of $f=0$ contain solutions of both $f_{1}=0$ and $f_{2}=0$.

The goal of this Section is to design an algorithm which for a given pair of quasilinear ordinary differential equations yields a quasi-linear common multiple. To simplify notations we assume that the equations are of first order: $y^{\prime}=p(y, x)$ and $y^{\prime}=q(y, x)$ where polynomials $p, q \in \mathbb{Q}[y, x]$, although one can extend the algorithm to equations of arbitrary orders almost literally.

Treating $y$ as a generic solution [9] of either of two given equations, one can assume that $y$ is algebraically independent of $x$ over $\mathbb{Q}$.

First, the algorithm looks for a common multiple being a quasi-linear second-order equation $y^{\prime \prime}=s\left(y^{\prime}, y, x\right)$ for a suitable polynomial $s(z, y, x) \in \mathbb{Q}[z, y, x]$. Hence

$$
s(p, y, x)=p^{\prime}=\frac{\partial p}{\partial y} \cdot p+\frac{\partial p}{\partial x}, s(q, y, x)=q^{\prime}=\frac{\partial q}{\partial y} \cdot q+\frac{\partial q}{\partial x} .
$$

Therefore

$$
s(z, y, x)=r \cdot(z-p)+\frac{\partial p}{\partial y} \cdot p+\frac{\partial p}{\partial x}=t \cdot(z-q)+\frac{\partial q}{\partial y} \cdot q+\frac{\partial q}{\partial x}
$$

for appropriate polynomials $r, t \in \mathbb{Q}[z, y, x]$, whence

$$
(t-r) \cdot(z-q)+r \cdot(p-q)=\left(\frac{\partial p}{\partial y} \cdot p+\frac{\partial p}{\partial x}\right)-\left(\frac{\partial q}{\partial y} \cdot q+\frac{\partial q}{\partial x}\right) .
$$

There exist $r, t \in \mathbb{Q}[z, y, x]$ which fulfil (7) iff

$$
(p-q) \mid\left(\frac{\partial p}{\partial y} \cdot p+\frac{\partial p}{\partial x}\right)-\left(\frac{\partial q}{\partial y} \cdot q+\frac{\partial q}{\partial x}\right) .
$$


If the latter relation holds, i. e. $r \cdot(p-q)=(p-q)^{\prime}$ for a suitable $r \in \mathbb{Q}[y, x]$, one can put $t:=r$ to get $(7)$ and take $s(z, y, x):=r \cdot(z-p)+p^{\prime}=r \cdot(z-q)+q^{\prime}$ to obtain a quasi-linear common multiple $y^{\prime \prime}=s\left(y^{\prime}, y, x\right)$. Conversely, when (7) holds, we substitute in it $z=q$. Thus, there exists a quasi-linear common multiple of the second order of a pair of equations $y^{\prime}=p(y, x), y^{\prime}=q(y, x)$ iff $(p-q)^{\prime} \in\langle p-q\rangle$, where $\langle p-q\rangle$ denotes the ideal generated by $p-q$.

More generally, following the same argument one can prove that

Lemma 2.2 There exists a quasi-linear common multiple of the order $n+1$ of a pair of first-order equations $y^{\prime}=p(y, x), y^{\prime}=q(y, x)$ iff $n$-th derivative

$$
(p-q)^{(n)} \in\left\langle p-q,(p-q)^{(1)}, \ldots,(p-q)^{(n-1)}\right\rangle .
$$

More explicitly, if the latter relation holds, i. e. $(p-q)^{(n)}=\sum_{0 \leq i<n} r_{i} \cdot(p-q)^{(i)}$ for some polynomials $r_{i} \in \mathbb{Q}[y, x], 0 \leq i<n$ then for polynomial

$$
s_{n}\left(z_{n}, \ldots, z_{1}, y, x\right):=\sum_{0 \leq i<n} r_{i} \cdot\left(z_{i+1}-p^{(i)}\right)+p^{(n)}=\sum_{0 \leq i<n} r_{i} \cdot\left(z_{i+1}-q^{(i)}\right)+q^{(n)}
$$

equation $y^{(n+1)}=s_{n}\left(y^{(n)}, \ldots, y^{\prime}, y, x\right)$ is a required quasi-linear common multiple.

For the proof we observe that $y^{(n+1)}=s_{n}\left(y^{(n)}, \ldots, y^{\prime}, y, x\right)$ for a polynomial $s_{n} \in$ $\mathbb{Q}\left[z_{n}, \ldots, z_{1}, y, x\right]$ is a common multiple iff

$$
s_{n}\left(p^{(n-1)}, \ldots, p, y, x\right)=p^{(n)}, s_{n}\left(q^{(n-1)}, \ldots, q, y, x\right)=q^{(n)} .
$$

Therefore,

$$
\begin{gathered}
s_{n}\left(z_{n}, \ldots, z_{1}, y, x\right)-p^{(n)} \in\left\langle z_{n}-p^{(n-1)}, \ldots, z_{1}-p\right\rangle, \\
s_{n}\left(z_{n}, \ldots, z_{1}, y, x\right)-q^{(n)} \in\left\langle z_{n}-q^{(n-1)}, \ldots, z_{1}-q\right\rangle .
\end{gathered}
$$

Subtracting two latter equalities we complete the proof of the lemma.

One can directly extend the lemma to a quasi-linear common multiple of a pair of quasi-linear equations of an arbitrary order.

Employing Hilbert's Idealbasissatz we obtain

Corollary 2.3 Any pair of ordinary quasi-linear differential equations has a quasi-linear common multiple.

Moreover, from the explicit bound on the Idealbasissatz [12] we obtain

Corollary 2.4 Any pair of ordinary quasi-linear differential equations

$$
y^{(k)}=p_{k}\left(y^{(k-1)}, \ldots, y, x\right), \quad y^{(k)}=q_{k}\left(y^{(k-1)}, \ldots, y, x\right)
$$


of order $k$ with polynomials of degrees $\operatorname{deg}\left(p_{k}\right), \operatorname{deg}\left(q_{k}\right) \leq d$ has a quasi-linear common multiple of order $g(d)$, where $g$ is a primitive-recursive function from the class $\mathcal{E}^{k+2}$ of Grzegorczyk's hierarchy [8], [15].

This provides also a complexity bound of the similar order of magnitude of the algorithm which looks for a quasi-linear common multiple by trying consecutively increasing orders of a candidate and solving the membership problem to an ideal (see Lemma 2.2), say, with the help of Gröbner basis. tially.

In particular, in case of first-order equations $(k=1)$ the function $g(d)$ grows exponen-

Example 2 Let $E_{1} \equiv y^{\prime}+y^{2}=0$ and $E_{2} \equiv y^{\prime}+y=0$. By Lemma 2.2 a multiple of order 2 does not exist; however, there is the following multiple of order 3 involving a parameter $C$ :

$$
E_{3} \equiv y^{\prime \prime \prime}+(C-4) y y^{\prime \prime}+(C+1) y^{\prime \prime}+(2 C-2) y^{\prime 2}+(2 C+2) y y^{\prime}+C y^{\prime}+C y^{2} .
$$

For $C=0$ it simplifies to

$$
E_{0} \equiv y^{\prime \prime \prime}+4 y y^{\prime \prime}+y^{\prime \prime}-2 y^{\prime 2}+2 y y^{\prime}=0 .
$$

Applying again Theorem 1.4 the factors $y^{\prime}+y^{2}, y^{\prime}+y$ and $y^{\prime}$ are obtained.

The next example is interesting because it allows to determine the general solution of all equations involved.

Example 3 Let $E_{1} \equiv y^{\prime}+y^{2}=0$ and $E_{2} \equiv y^{\prime}=0$ with solutions $y=\frac{1}{x+C}$ and $y=C$ respectively. The multiple of $E_{1}$ and $E_{2}$ yields $y^{\prime \prime}+2 y y^{\prime}=0$ with first integral $y^{\prime}+y^{2}=C$. Its general solution is $y=C_{1} \tan \left(C_{2}-C_{1} x\right)$. It is not obvious how the latter solution is related to the two solutions involving a single parameter.

Remark 2.5 The general solution of the second-order equation in the preceding example may also be written as $y=C_{1} \tanh \left(C_{2}+C_{1} x\right)$; the two representations are transformed into each other by multiplying the constants with the complex unit $i$ and representing them in terms of exponentials. From the latter representation the constant solution may be obtained by taking the limit $C_{2} \rightarrow \infty$. The first integral $y^{\prime}+y^{2}=C$ generalizes the divisor $E_{1}$; its existence simplifies the solution procedure because it provides already one of the constants involved in the general solution of the second-order equation.

Acknowledgements. The first author is grateful to the Max-Planck Institut für Mathematik, Bonn for its hospitality during writing this paper and to Labex CEMPI (ANR-11LABX-0007-01). 


\section{References}

[1] X.-S. Gao, M. Zhang, Decomposition of ordinary differential polynomials, Appl. Alg. Eng. Commun. Comput. 19 (2008), 1-25.

[2] E. Goursat, Leçon sur l'intégration des équation aux dérivées partielles. vol. II, A. Hermann, Paris, 1898.

[3] D. Grigoriev, Complexity of factoring and GCD calculating of ordinary linear differential operators, J. Symp. Comput. 10 (1990), 7-37.

[4] D. Grigoriev, Analogue of Newton-Puiseux series for non-holonomic D-modules and factoring, Moscow Math. J. 9 (2009), 775-800.

[5] D. Grigoriev, F. Schwarz, Factoring and solving linear partial differential equations, Computing 73 (2004), 179-197.

[6] D. Grigoriev, F. Schwarz, Loewy and primary decompositions of D-modules, Adv. Appl. Math. 38 (2007), 526-541.

[7] D. Grigoriev, F. Schwarz, Non-holonomic ideal in the plane and absolute factoring, Proc. Intern. Symp. Symbol. Algebr. Comput., ACM, Munich (2010), 93-97.

[8] A. Grzegorczyk, Some classes of recursive functions, Rozprawy Matematiczne 4, (1953), 1-44.

[9] E. Kolchin, Differential algebra and algebraic groups, Academic Press, 1973.

[10] L. Schlesinger, Handbuch der Theorie der linearen Differentialgleichungen II, Teubner, 1897.

[11] F. Schwarz, A Factorization Algorithm for Linear Ordinary Differential Equations, Proc. ACM Intern. Symp. Symbol. Algebr. Comput. (1989), 17-25.

[12] A. Seidenberg. On the length of a Hilbert ascending chain. Proc. Amer. Math. Soc. 29 (1971), 443-450.

[13] S. Tsarev, On factorization of nonlinear ordinary differential equations, Proc. ACM Symp. Symbol. Algebr. Comput., Vancouver, (1999), 159-164.

[14] S. Tsarev, Generalized Laplace transformations and integration of hyperbolic systems of linear partial differential equations, Proc Intern. Symp. Symbol. Algebr. Comput., ACM, Peking, (2005), 325-331

[15] K. Wagner, G. Wechsung. Computational Complexity. Mathematics and its Applications 21, 1986. 